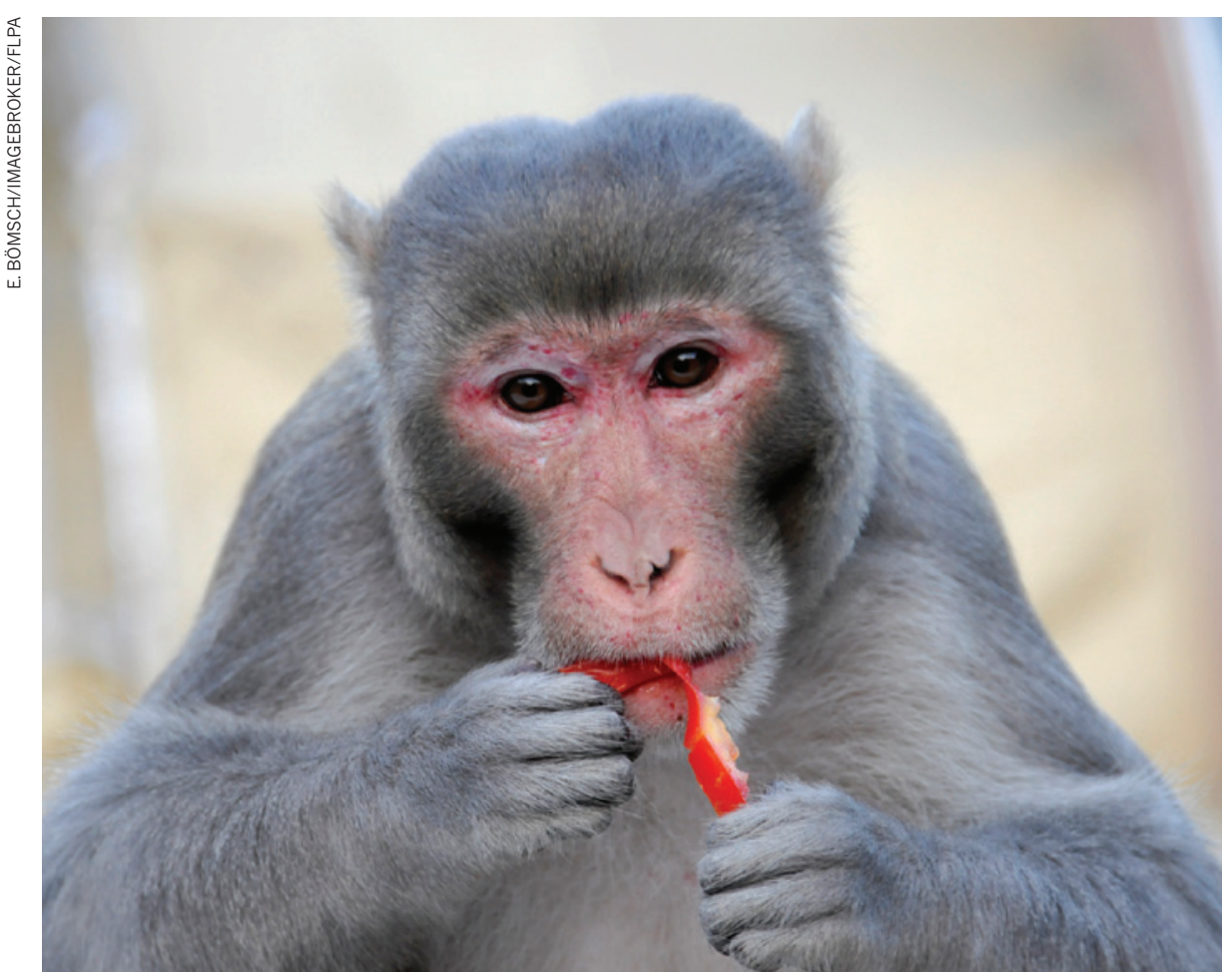

Rhesus monkeys on calorie-restricted diets age just as quickly as their chubbier counterparts.

AGEING

\title{
Calorie restriction falters in the long run
}

Genetics and healthy diets matter more for longevity.

\section{BY AMY MAXMEN}

$\mathrm{T}$ o those who enjoy the pleasures of the dining table, the news may come as a relief: drastically cutting back on calories does not seem to lengthen lifespan in primates.

The verdict, from a 25-year study in rhesus monkeys fed $30 \%$ less than control animals, represents another setback for the notion that a simple, diet-triggered switch can slow ageing. Instead, the findings, published this week in Nature ${ }^{1}$, suggest that genetics and dietary composition matter more for longevity than a simple calorie count.

"To think that a simple decrease in calories caused such a widespread change, that was remarkable," says Don Ingram, a gerontologist at Louisiana State University in Baton Rouge, who designed the study almost three decades ago while at the National Institute on Aging (NIA) in Bethesda, Maryland.

When the NIA-funded monkey study began, however, studies of caloric restriction in shortlived animals were hinting at a connection. Experiments had showed that starvation made roundworms live longer. Other studies had showed that rats fed fewer calories than their slow and balding brethren maintained their shiny coats and a youthful vigour. And more recently, molecular studies had suggested that caloric restriction - or compounds that mimicked it - might trigger a cascade of changes in gene expression that had the net effect of slowing ageing.

In 2009, another study ${ }^{2}$, which began in 1989 at the Wisconsin National Primate Research Center (WNPRC) in Madison, concluded that caloric restriction did extend life in rhesus monkeys. The investigators found that $13 \%$ of the dieting group died from age-related causes, compared with $37 \%$ of the control group.

One reason for that difference could be that the WNPRC monkeys were fed an unhealthy diet, which made the calorie-restricted monkeys seem healthier by comparison simply because they ate less of it. The WNPRC monkeys' diets contained $28.5 \%$ sucrose, compared with

\section{$\rightarrow$ NATURE.COM} Read the News \& Views article about this research. go.nature.com/icodos
$3.9 \%$ sucrose at the NIA. Meanwhile, the NIA meals included fish oil and antioxidants, whereas the WNPRC meals did not. Rick Weindruch, a gerontologist at the WNPRC who led the study, admits: "Overall, our diet was probably not as healthy."

Further, the WNPRC control group probably ate more overall, because their meals were unlimited, whereas NIA monkeys were fed fixed amounts. As adults, control monkeys in the WNPRC study weighed more than their NIA counterparts. Overall, the WNPRC results might have reflected an unhealthy control group rather than a long-lived treatment group. "When we began these studies, the dogma was that a calorie is a calorie," Ingram says. "I think it's clear that the types of calories the monkeys ate made a profound difference."

Researchers studying caloric restriction in mice have become accustomed to mixed results, which they attribute to genetic diversity among strains. Genetics probably explains part of the variation between the monkey studies, too, as the NIA monkeys were descended from lines from India and China, whereas the Wisconsin monkeys were all from India.

The molecular effects of caloric restriction have also turned out to be complicated. Using compounds such as resveratrol, found in red wine, scientists have triggered the stress response that caloric restriction activates, which shuts down non-vital processes in favour of those that ward off disease. But hopes that ageing could be delayed by targeting a single gene or protein in a single molecular pathway have faded, as researchers have learned that the key pathways vary according to the animal."It may take us a decade to sort out longevity networks," says David Sinclair, a geneticist at Harvard Medical School in Boston, Massachusetts.

Meanwhile, there is a dearth of evidence that caloric restriction slows ageing in humans. Observational studies have found that people of average weight tend to live longest ${ }^{3}$. Nir "Warzilai, a gerontolo"When we began gist at Albert Einstein these studies, the dogma was that a calorie is acalorie."

College of Medicine in New York, says that the centenarians he studies have led him to believe that genetics is more important than diet and lifestyle. "They're a chubby bunch," he says.

A more nuanced picture would suit Ingram, who enjoys an occasional feast of Louisiana crawfish. Ingram says that he looks forward to studies of how diet composition, rather than caloric intake, affects ageing. "Is the human lifespan fixed?" he asks. "I still don't believe that for a minute."
1. Mattison, J. A et al. Nature http://dx doi. org/10.1038/nature11432 (2012)

2. Colman, R. J. et al. Science 325, 201-204 (2009)

3. Berrington de Gonzalez, A. et al. N. Engl. J. Med. 363, 2211-2219 (2010). 